\title{
Adaptive Multiscale Material Characterization for Multi-scale Modeling of Aluminum Alloys
}

\author{
D. M. Valiveti ${ }^{*}$ and S. Ghosh \\ * Graduate Research Associate, Department of Mechanical Engineering, The Ohio State \\ University, Columbus, OH 43210, USA \\ I John B. Nordholt Professor, Department of Mechanical Engineering, The Ohio State University, \\ Columbus, $\mathrm{OH} 43210$, USA
}

Heterogeneous materials like cast aluminum alloys containing silicon and intermetallics, are commonly used in automotive, aerospace and other industries. The different phases and inclusions (figure 1a) in these materials have a significant effect on the material behavior and failure properties. Many experimental studies have shown that their tensile and fracture behavior are affected by their microstructure, e.g. shape and spatial distributions [1]. Effective modeling of fracture and crack propagation requires comprehensive analysis of large domains of material and the notion of multiscale modeling is vital for information transfer between various scales of the problem. Various multiple scale models have been proposed in the literature, e.g. in [2,3]. These models require detailed modeling at the microstructural scale with careful delineation of the morphology. However these computations are very intensive and cannot be conducted at all locations of the macro model. Thus a systematic characterization of the material morphology is necessary at various scales to delineate regions where microstructural modeling is critical and where it is not, from a macroscopic modeling point of view. This effort is also needed for transfer of microstructural information to the other scales of the model.

Microstructural information is necessary at a large number of points in the macro domain for multiscale modeling of failure in the Al alloys,. A wide variety of methods have been introduced in literature for image analysis and microstructural characterization $[4,5,6]$. Some of these involve tessellation of the microstructure and image analysis while others have evaluated point statistics by grabbing contiguous images followed by digital image montage. In this paper an adaptive multiscale characterization method, involving a medley of image processing (compression and rarefaction), microstructure characterization using statistics and sampling and adaptive refinement is proposed for generating a hierarchical computational model that will precede computational modeling of response and failure. It deals with characterization for application to micromechanics modeling, taking care that the parameters evaluated are appropriate for modeling complex material microstructures. Characterization is conducted at different magnifications to evaluate properties of the material at respective magnifications. The two point correlation functions (figure 1b), mark correlation functions and coefficient of variance [4] are used for characterizing the length scales. A novel procedure is also proposed to obtain the microstructure of the entire domain from sample high resolution microstructures at selected areas. For the adaptive delineation of different scales, required in the modeling process discretization and level change functionals are defined based on microstructure characteristics. Finally a three level multiscale discretization is demonstrated with various functionals derived in the study (see figure 2). The paper will conclude with a multi-scale modeling exercise demonstrating the effectiveness of the proposed multi-scale characterization for the study of failure in heterogeneous materials. 
References

[1] Q.G. Wang, C.H. Caceres, J. R. Griffiths.: Metall. Mater. Trans. A, 2003, 34A, pp.2901-2912.

[2] P. Raghavan and S. Ghosh: Comp. Model. Engng. Sci., 2004, 5, pp.151-170.

[3] Z. Shan and A. M. Gokhale, Intl. Jour. Plasticity, 2004, 20, pp. 1347-1370.

[4] M. Li, Ghosh, S. and O. Richmond, : Mat. Sci. Engg., 1999, A266, pp.221-240

[5] A. Tewari, A. M. Gokhale, J. M.Spowart, D. B. Miracle: Act. Mater. ,2004, 52, pp. 307-319.

[6] A. M. Gokhale, S. Yang, Metall. Mater. Trans., 1999, A30, pp. 2369-2381.

The authors gratefully acknowledge financial support for this work from the National Science Foundation through a GOALI grant No. CMS 0308666.
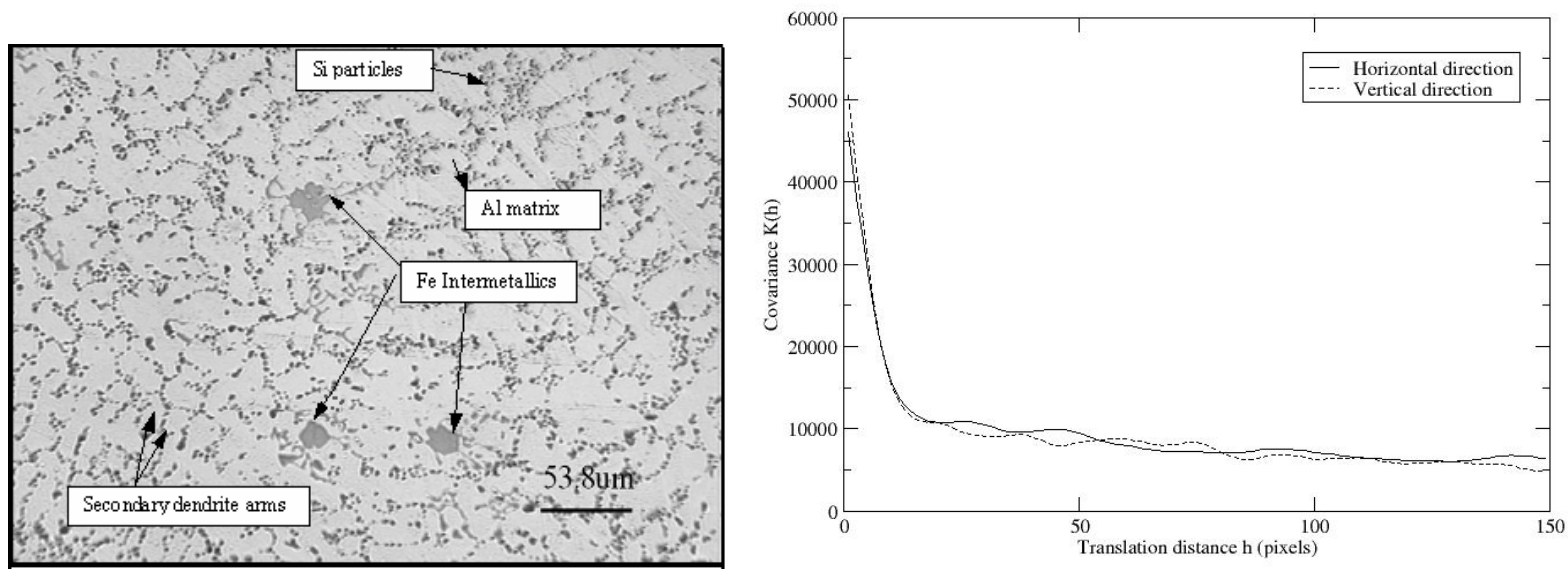

Figure 1. (a) SEM image of a cast aluminum microstructure (b) covariance function of the microstructure in two orthogonal direction
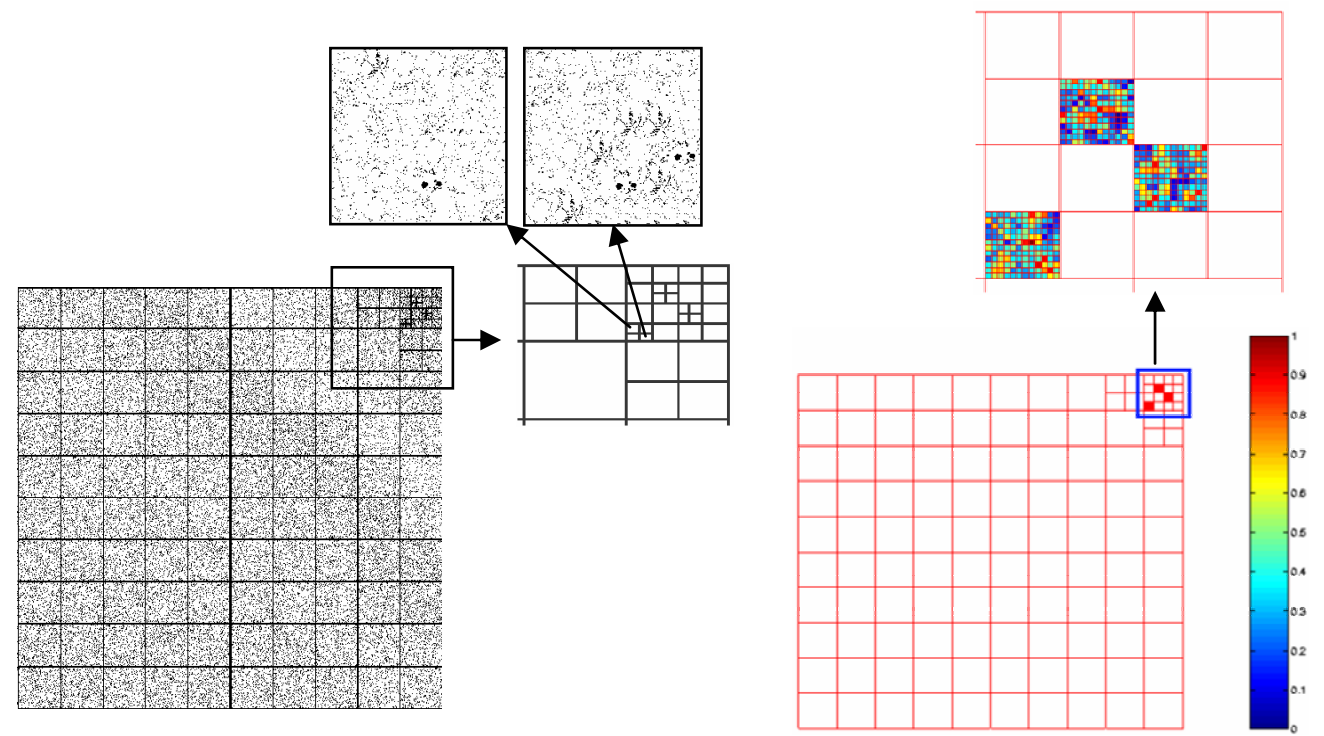

Figure 2 : Adaptive domain decomposition based on microstructural characterization and contour plot of level change functional. 